\title{
ROTA AMBIENTAL: LEVANTAMENTO FLORÍSTICO DA VEGETAÇÃO EMPREGADA NA ARBORIZAÇÃO DA UNIVATES, LAJEADO/RS
}

\section{RESUMO}

\author{
Jonas Bernardes Bica1; Cátia Viviane Gonçalves²; André Jasper ${ }^{3} 4$
}

A integração da sociedade com o meio ambiente, muitas vezes passa desapercebida no cotidiano de transeuntes que circulam por áreas verdes, por outro lado, o paisagismo urbano busca harmonia entre o ambiente natural e o construído. Buscou-se identificar as espécies nativas e exóticas encontradas nas áreas de uso comum do campus da Univates. Foram coletadas amostras de espécies arbóreas e arborescentes presentes nas áreas comuns do campus e, com auxílio de chaves dicotômicas específicas, realizou-se a identificação das mesmas. Foram encontradas 465 espécimes de 59 espécies, distribuídas em 26 famílias. As espécies exóticas tiveram uma maior ocorrência do que as nativas, sendo que foram encontradas 35 (50,3\%) espécies exóticas e 27 (49,7\%) nativas. As espécies encontradas com maior frequência foram Dypsis lutescens (65 indivíduos), Syagrus romanzoffiana (52 indivíduos), Inga marginata (77 indivíduos), Peltophorum dubium (21 indivíduos) e Bauhinia sp. (27 indivíduos). As espécies Butia capitata, Ficus cestrifolia e Ficus luschnathiana são destaque por sua importância ecológica. Visando proporcionar a maior interação entre os estudantes e o meio ambiente, foram implantadas placas com informações sobre ocorrência, nomenclatura científica e popular das espécies, contribuindo para conhecimento monitorado ou autoguiado bem como para a valorização e auxílio na manutenção dos ambientes verdes da Univates.

Palavras-chave: Áreas verdes; identificação arbórea; interação; conhecimento autoguiado.

\section{ENVIRONMENTAL ROTE: A FLORISTIC EMPLOY OF THE VEGETATION USED AT THE UNIVATES AFFORESTATION, LAJEADO/RS}

\section{ABSTRACT}

The integration of society with the environment, often goes unnoticed in the daily circulating passersby green areas, on the other hand, urban landscaping seeks harmony between the natural and built environment. We aimed to identify native and exotic species found in the Univates campus common areas. Samples of the arborescent tree species from the common areas of the campus were collected and, with the aid of specific dichotomous keys, held their identification. We found 465 specimens of 59 species in 26 families. Exotic species had a higher incidence than the native, and 35 (50.3\%) exotic species and 27 (49.7\%) native species were found. The most frequently species found were Dypsis lutescens (65 individuals), Syagrus romanzoffiana (52 individuals), Inga marginata (77 individuals), Peltophorum dubium (21 individuals) and Bauhinia sp. (27 individuals). The species Butia capitata, Ficus luschnathiana and Ficus cestrifolia are highlighted by its ecological significance. Aiming to providing a greater interaction between the students and the environment, informational signs with data about the occurrence, scientific nomenclature and popular for knowledge or self-guided and monitored for recovery were implanted. That also aid to preserve the green environment idea at Univates.

Keywords: green areas; tree identification; interaction; self-guided knowledge.

\footnotetext{
${ }^{1}$ Biólogo, Mestrando do Programa de Pós-graduação em Ambiente e Desenvolvimento do Centro Universitário UNIVATES (PPGAD/Univates - Bolsa FAPERGS), Lajeado, Rio Grande do Sul, contato: jonas2bel@universo.univates.br

${ }^{2}$ Bióloga, Mestre em Ecologia, coordenadora do Programa Interno de Separação de Resíduos do Centro Universitário UNIVATES (PISR/UNIVATES), Lajeado, Rio Grande do Sul, contato: biologacatia@univates.br

3 Biólogo, Doutor em Geociências, Docente do Programa de Pós-graduação em Ambiente e Desenvolvimento do Centro Universitário UNIVATES (PPGAD/Univates), Bolsista de Produtividade em Pesquisa CNPq, Lajeado, Rio Grande do Sul, contato: ajasper@univates.br

${ }^{4}$ Recebido em 30.09.2013 e aceito para publicação em 15.12.2013
} 
A arborização urbana contribui para a manutenção de um ambiente urbano agradável tendo influência decisiva na qualidade de vida nas cidades e, portanto, na saúde da população (MÜLLER, 1998). Shams; Giacomeli e Sucomine (2009) afirmam que uma urbanização acelerada, pode incitar sérios danos à condição ambiental, bem como consideráveis prejuízos econômicos, sociais e de qualidade de vida às comunidades urbanas. Neste sentido, além da qualidade de vida das pessoas, a arborização urbana, se faz muito importante também na função da conservação da biodiversidade no meio urbano (RICHTER et al., 2012).

Sanchotene (1994) definiu a arborização urbana como sendo o conjunto da vegetação arbórea, natural ou cultivada, situada em áreas particulares, parques, praças, vias públicas e em áreas verdes complementares. Richter et al. (2012) afirmam que a arborização urbana diz respeito aos elementos vegetais de porte arbóreo, dentro da cidade, neste contexto, as árvores plantadas em calçadas, fazem parte desta composição paisagística, assim como parques e praças. Segundo Schanzer (2003), a presença da vegetação exerce grande influência em aspectos de conforto ambiental principalmente nas questões referentes ao conforto térmico e a percepção do ambiente construído.

Trabalhos de levantamentos florísticos de campi permitem o desenvolvimento de ações de manejo e monitoramento para a arborização local, o que propicia benefícios ambientais e paisagísticos, além do bem-estar à população que circula na área (COSTA; MACHADO, 2009), valorizando a arborização existente em cada local (KURIHARA; IMAÑA-ENCINAS; PAULA, 2005).

No sentido de promover o conhecimento popular e científico este trabalho foi desenvolvido com o objetivo de identificar as espécies arbóreas e arborescentes nas áreas comuns do campus.

\section{MATERIAIS E MÉTODOS}

O campus do Centro Universitário UNIVATES

(Figura 1), encontra-se localizado no município de Lajeado, no Vale do Taquari, Rio Grande do Sul, e está inserido no bioma Mata Atlântica, integrando a região fitogeográfica da Floresta Estacional Decidual.

Foram identificadas as espécies arbóreas e arborescentes encontradas nas áreas comuns de circulação da instituição. As espécies nativas foram identificadas com auxílio de chave dicotômica proposta por Sobral et al. (2013). Para isso, o material vegetal foi coletado, seguindo-se o proposto por Jasper, Musskopf e Majolo (1997). As espécies exóticas foram identificadas com auxílio de bibliografia para espécies exóticas do Rio Grande do Sul, principalmente Lorenzi (2003). 
Figura 1. Vista aérea do campus da Univates.

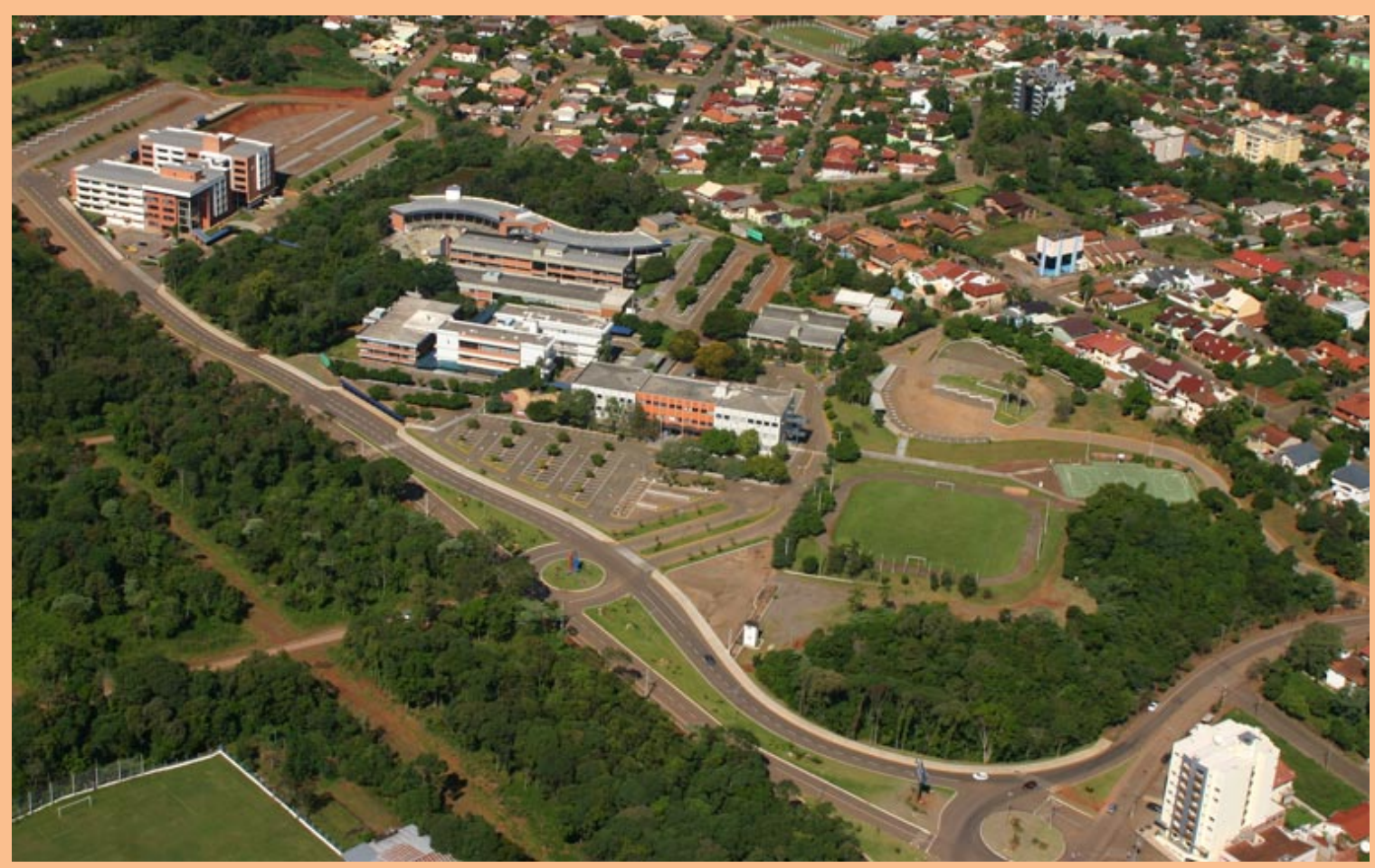

Fonte: http://www.univates.br/files/files/univates/institucional/univates_aerea_1_12.jpg, consultado em

26 de fevereiro de 2012

Depois de identificadas, todos os espécimes registrados receberam placas informativas contendo as seguintes informações: família; nome científico e nome popular. As espécies protegidas pelas Leis $n^{\circ}$ 11026/97 e 9519/92 do estado do Rio Grande do Sul, além das placas de identificação, receberam ainda placas específicas indicando sua importância legal.

\section{RESULTADOS E DISCUSSÃO}

Foram identificados e demarcados 465 espécimes de 59 espécies e 25 famílias (Tabela

1). As espécies exóticas apresentaram uma maior diversidade em relação às espécies nativas, sendo encontradas 35 espécies exóticas e 27 nativas.

Tabela 1. Espécies encontradas no campus do Centro Universitário Univates divididos em famílias e suas respectivas quantidades

\begin{tabular}{lllccc}
\hline \multicolumn{1}{c}{ Família } & \multicolumn{1}{c}{ Nome científico } & Nome popular & $\begin{array}{c}\text { Nr. de } \\
\text { espécimes } \\
\text { encontrados }\end{array}$ & $\begin{array}{c}\text { F. } \\
\text { relativ } \\
\text { a }\end{array}$ & $\begin{array}{c}\text { Ocorrênci } \\
\text { a }\end{array}$ \\
\hline $\begin{array}{l}\text { Anacardiacea } \\
\text { e }\end{array}$ & Manguifera indica & Manga & 1 & $0,21 \%$ & Exótica \\
& Schinus terebinthifolius & Aroeira- & 15 & $3,22 \%$ & Nativa \\
\hline
\end{tabular}


vermelha

\begin{tabular}{|c|c|c|c|c|c|}
\hline Araliaceae & Schefflera arborícola & Cheflera & 1 & $0,21 \%$ & Exótica \\
\hline Araucariaceae & Araucaria columnaris & $\begin{array}{l}\text { Pinheiro-de- } \\
\text { natal }\end{array}$ & 1 & $0,21 \%$ & Exótica \\
\hline \multirow{6}{*}{ Arecaceae } & $\begin{array}{l}\text { Archontophoenix } \\
\text { cunninghamii }\end{array}$ & Palmeira-real & 5 & $1,07 \%$ & Exótica \\
\hline & Butia capitata & Butiá & 6 & $1,29 \%$ & Nativa \\
\hline & Dypsis decary & $\begin{array}{l}\text { Palmeira- } \\
\text { triangular }\end{array}$ & 8 & $1,72 \%$ & Exótica \\
\hline & Dypsis lutescens & Areca-bambu & 65 & $13,98 \%$ & Exótica \\
\hline & Phoenix roebelinii & Tamareira-anã & 12 & $2,58 \%$ & Exótica \\
\hline & Syagrus romanzoffiana & Gerivá & 52 & $11,18 \%$ & Nativa \\
\hline Asteraceae & Annona sylvatica & Araticum & 1 & $0,21 \%$ & Nativa \\
\hline \multirow{4}{*}{ Bignoniaceae } & $\begin{array}{l}\text { Handroanthus } \\
\text { crisotrichus }\end{array}$ & Ipê-amarelo & 19 & $4,09 \%$ & Exótica \\
\hline & $\begin{array}{l}\text { Handroanthus } \\
\text { heptaphylus }\end{array}$ & Ipê-roxo & 9 & 1,93\% & Nativa \\
\hline & Jacaranda micrantha & Caroba & 1 & $0,21 \%$ & Nativa \\
\hline & Jacaranda mimosifolia & $\begin{array}{l}\text { Jacarandá- } \\
\text { mimoso }\end{array}$ & 5 & $1,07 \%$ & Exótica \\
\hline Cannabaceae & Trema micrantha & Grandiúva & 3 & $0,64 \%$ & Nativa \\
\hline \multirow{4}{*}{ Cupressaceae } & Chamaecyparis obtusa & $\begin{array}{l}\text { Cipreste- } \\
\text { dourado }\end{array}$ & 1 & $0,21 \%$ & Exótica \\
\hline & Cupressus lusitanica & $\begin{array}{l}\text { Cipreste- } \\
\text { português }\end{array}$ & 15 & $3,22 \%$ & Exótica \\
\hline & Cupressus sempervirens & Cipreste-comum & 1 & $0,21 \%$ & Exótica \\
\hline & $\begin{array}{l}\text { Juniperus chinensis } \\
\text { torulosa }\end{array}$ & Kaizuca & 1 & $0,21 \%$ & Exótica \\
\hline Cycadaceae & Cycas revoluta & $\begin{array}{l}\text { Palma-de-santa- } \\
\text { rita }\end{array}$ & 3 & $0,64 \%$ & Exótica \\
\hline \multirow{2}{*}{$\begin{array}{l}\text { Euphorbiacea } \\
\text { e }\end{array}$} & Alchornea triplinervia & Tanheiro & 4 & $0,86 \%$ & Nativa \\
\hline & Sapium glandulosum & Leiteiro & 3 & $0,64 \%$ & Nativa \\
\hline \multirow{10}{*}{ Fabaceae } & Acacia mearnsii & Acacia & 1 & $0,21 \%$ & Exótica \\
\hline & Bauhinea sp. & Pata-de-vaca & 26 & $5,59 \%$ & Exótica \\
\hline & Caesalpinia ferrea & Pau-ferro & 3 & $0,64 \%$ & Nativa \\
\hline & $\begin{array}{l}\text { Caesalpinia } \\
\text { peltophoroides }\end{array}$ & Sibipiruna & 12 & $2,58 \%$ & Exótica \\
\hline & Calliandra brevipes & $\begin{array}{l}\text { Topete-de- } \\
\text { cardeal }\end{array}$ & 1 & $0,21 \%$ & Nativa \\
\hline & Inga marginata & Ingazeiro & 77 & $16,56 \%$ & Nativa \\
\hline & Peltophorum dubium & Canafístula & 21 & $4,52 \%$ & Nativa \\
\hline & Schizolobium parahyba & Guapuruvu & 1 & $0,21 \%$ & Exótico \\
\hline & Senna oblongifolia & Acácia & 6 & $1,29 \%$ & Nativa \\
\hline & Tipuana tipu & Tipuana & 6 & $1,29 \%$ & Exótica \\
\hline \multirow[t]{4}{*}{ Juglandaceae } & Juglans regia & Nogueira & 1 & $0,21 \%$ & Exótica \\
\hline & Cinnamomun verun & Canela-da-índia & 5 & $1,07 \%$ & Exótica \\
\hline & Nectandra oppositifolia & $\begin{array}{l}\text { Canela- } \\
\text { ferrugem }\end{array}$ & 1 & $0,21 \%$ & Nativa \\
\hline & Persea americana & Abacateiro & 2 & $0,43 \%$ & Exótica \\
\hline \multirow[t]{3}{*}{ Meliaceae } & Guarea machrophylla & $\begin{array}{l}\text { Catiguá- } \\
\text { morcego }\end{array}$ & 1 & $0,21 \%$ & Nativa \\
\hline & Melia azedarach & Cinamomo & 1 & $0,21 \%$ & Exótica \\
\hline & Ficus benjamini & Fico-chorão & 3 & $0,64 \%$ & Exótica \\
\hline \multirow[t]{2}{*}{ Moraceae } & Ficus cestrifolia & $\begin{array}{l}\text { Figueira-de- } \\
\text { folha-miúda }\end{array}$ & 3 & $0,64 \%$ & Nativa \\
\hline & Ficus luschnathiana & Figueira & 2 & $0,43 \%$ & Nativa \\
\hline
\end{tabular}

Jonas Bernardes Bica et al. 


\begin{tabular}{|c|c|c|c|c|c|}
\hline \multirow{6}{*}{ Myrtaceae } & $\begin{array}{l}\text { Campomanesia } \\
\text { xanthocarpa }\end{array}$ & Guabiroba & 2 & $0,43 \%$ & Nativa \\
\hline & Eugenia involucrata & Cerejeira & 18 & $3,87 \%$ & Nativa \\
\hline & Eugenia pyriformis & Uvaia & 1 & $0,21 \%$ & Nativa \\
\hline & Eugenia uniflora & Pitangueira & 4 & $0,86 \%$ & Nativa \\
\hline & Psydium guajava & Goiabeira & 12 & $2,58 \%$ & Exótica \\
\hline & Sizygium jambolana & João-bolão & 3 & $0,64 \%$ & Exótico \\
\hline $\begin{array}{l}\text { Nyctaginacea } \\
\mathrm{e}\end{array}$ & Bougainvillea spectabilis & Três-marias & 3 & $0,64 \%$ & Exótica \\
\hline Oleaceae & Ligustrum lucidum & Ligustro & 5 & $1,07 \%$ & Exótica \\
\hline Pinaceae & Pinus eliottii & $\begin{array}{l}\text { Pinheiro- } \\
\text { americano }\end{array}$ & 1 & $0,21 \%$ & Exótico \\
\hline Proteaceae & Grevillea robusta & Grevilea & 1 & $0,21 \%$ & Exótica \\
\hline Rhamnaceae & Hovenia dulcis & Uva-do-japão & 1 & $0,21 \%$ & Exótica \\
\hline Rosaceae & Eryobotria japonica & Nespereira & 1 & $0,21 \%$ & Exótica \\
\hline Salicaceae & Casearia sylvestris & Chá-de-bugre & 3 & $0,64 \%$ & Nativa \\
\hline \multirow{3}{*}{ Sapindaceae } & Allophilus edulis & Chau-chau & 2 & $0,43 \%$ & Nativa \\
\hline & Cupania vernalis & $\begin{array}{l}\text { Camboatá- } \\
\text { vermelho }\end{array}$ & 1 & $0,21 \%$ & Nativa \\
\hline & Matayba juglandifolia & $\begin{array}{l}\text { Camboatá- } \\
\text { branco }\end{array}$ & 1 & $0,21 \%$ & Nativa \\
\hline Solanaceae & Solanum mauritianum & Fumo-bravo & 1 & $0,21 \%$ & Nativa \\
\hline
\end{tabular}

As espécies nativas representam 52,04\% dos espécimes encontrados (242 espécimes) e 47,96\% os espécimes exóticos (223 espécimes). As espécies encontradas com maior frequência relativa foram Inga marginata, 16,56\%, representada por 77 indivíduos, Dypsis lutescens, 13,98\%, representada por 65 indivíduos, Syagrus romanzoffiana, 11,18\%, representada por 52 indivíduos. Tais resultados vão ao encontro do que é defendido por Batista et al. (2013), pois afirmam que é possível selecionar espécies nativas regionais adequadas para o enriquecimento da diversidade da arborização urbana de ruas, praças e avenidas. Para Melo e Severo (2007) a utilização de plantas ornamentais com o intuito paisagístico, como composição cênica ou de destaque/realce de estruturas e ambientes vem, com o passar dos anos, aliando-se a aspectos funcionais da utilização desta mesma vegetação com múltiplos objetivos. A arte do belo, à estética e à forma, agrega-se o aspecto funcional, a simbiose entre o objetivo e o objeto, em que o foco vem a ser a melhoria da qualidade de vida da população (ANGELIS NETO; ANGELIS, 1999).

Constatou-se ainda a preservação das espécies Butia capitata, Ficus cestrifolia e Ficus luschnathiana, identificadas no campus e que são destaque por sua importância ecológica, além de serem protegidas pelas Leis $n^{\circ}$ 11026/97 e 9519/92 do estado do Rio Grande do Sul, sendo identificadas com placa específica (Figura 2) e mantidas em locais estratégicos quanto ao interesse ecológico e paisagístico. 
Figura 2. Placa específica para os espécimes protegidos por Lei Estadual.

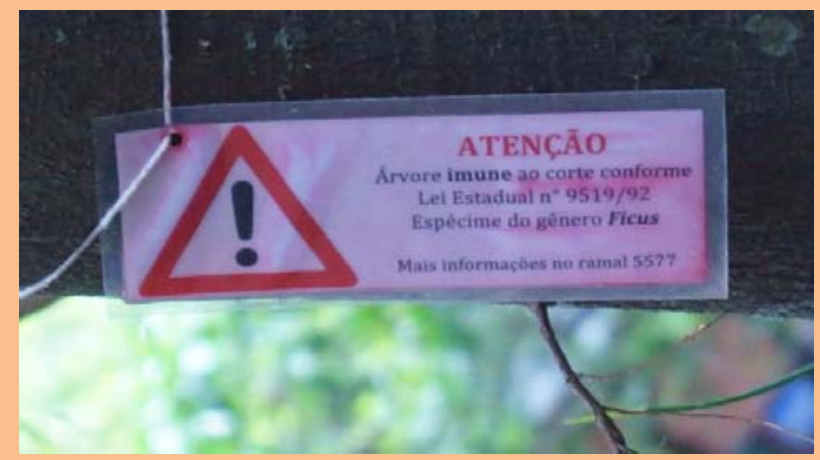

Fonte: Setor de Botânica e Paleobotânica da UNIVATES.

A vegetação do campus, segundo Melo e Severo

(2007), pode despertar nos usuários a consciência ambiental, através da interpretação da natureza e da vivência em áreas mais preservadas e com biodiversidade paisagística. Alcançando, neste sentido, o objetivo de uma sociedade mais integrada com o meio ambiente e, por sua vez, compreendendo a importância de tais recursos paisagísticos, tanto para as interações ecológicas, quanto para o bem estar da sociedade.

\section{CONCLUSÃO}

Com a realização do presente estudo foram observados 465 espécimes arbóreos e arborescentes nas áreas comuns do campus. Através da demarcação e caracterização destes espécimes, possibilitou-se levar ao conhecimento dos estudantes e visitantes da Univates a diversidade vegetal arbórea presente no campus.

Através dos meios de comunicação da Universidade, busca-se, atualmente, promover a interação e contato dos estudantes e visitantes na UNIVATES com o ambiente natural das áreas comuns do campus, além de divulgar as áreas verdes da instituição, como uma forma de acessibilidade à informação das interações da academia com a comunidade, sendo esta, uma forma de proporcionar o conhecimento popular e o conhecimento científico à toda comunidade.

\section{AGRADECIMENTO}

Aos acadêmicos do curso de Ciências Biológicas do Centro Universitário UNIVATES Eduardo Graf, Rosane Pereira da Silva,
Claudete Teresinha Klafke Mallman, Talyssa Valerius e Joana Beuren. 
Jonas Bernardes Bica agradece à FAPERGS pela concessão de bolsa de mestrado. André Jasper agradece ao auxílio financeiro do CNPq,
CAPES e FAPERGS, por meio de apoio à pesquisa e bolsa de produtividade em pesquisa.

\section{REFERÊNCIAS}

ANGELIS NETO, G; ANGELIS, B.L.D. de Plantas ornamentais: do paisagismo a outras aplicações. Rev. Bras. Hortc. Ornam., Campinas, v.5, n.1, p.12-19, 1999.

BATISTA, M. L.; LOPES, A. G.; DE CARVALHO, F. A.; DA SILVA, L. F.; GALVES, N.; PEDRO, N. F.; STRANGHETTI, V. Indicação de essências regionais do noroeste paulista para enriquecimento da arborização de ruas, praças e avenidas. REVSBAU, Piracicaba - SP, v.8, n.1, p.75-88, 2013.

COSTA, I.S.; MACHADO, R.R.B. A arborização do câmpus da UESPI Poeta Torquato Neto em Teresina (PI): diagnóstico e monitoramento. REVSBAU, v. 4, n.4, p.32-46, 2009.

JASPER, A.; MUSSKOPF, E. L.; MAJOLO, M. A. Manual para preparação de material de apoio didático para aulas práticas na área de ciências e biologia. Lajeado: Univates, 1997.

KHALIL, M. A. K. Genetic improvement of trees for the urban environment: challenges and opportunities. In: CANADIAN TREE IMPROVEMENT ASSOCIATION SYMPOSIUM, 16, Ottawa, 1997. Proceedings. Ottawa, Canadian Forestry Service. p. 09-19.

KURIHARA, D. L.; IMAÑA-ENCINAS, J.; PAULA, J. E. Levantamento da arborização do câmpus da Universidade de Brasília. Cerne, v. 11, n.2, p.127-36, 2005.

LORENZZI, H.; DE SOUZA, H. M.; TORRES, M. A. V.; BACHER, L. B. Árvores exóticas no Brasil: madeireiras, ornamentais e aromáticas. Nova Dessa: Instituto Plantarum, 2003.

MAO, C. C. M. Arborização urbana - Projeto básico para Três Passos - RS. In: II Congresso Brasileiro de Arborização Urbana; V Encontro Nacional sobre Arborização Urbana. Anais... São Luiz: SBAU, 1994. p.403-419.

MÜLLER, J. Orientação básica para manejo da arborização urbana. Edições FAMURS. Porto Alegre: Nova Prova, 1998.

MELO, E. F. R. Q.; SEVERO, B. M. A. Vegetação arbórea no câmpus da Universidade de Passo Fundo. Revista da Sociedade Brasileira de Arborização Urbana, v.2, n. 2, p. 76-87, 2007.

RICHTER, C.; PEITER, M. X.; ROBAINA, A. D.; DE SOUZA, A. R. C.; FERRAZ, R. C.; DE DAVID, A. F. Levantamento da arborização urbana pública de Mata/RS. REVSBAU, Piracicaba SP, v.7, n.3, p.88-96, 2012.

SANCHOTENE, M. C. C. Desenvolvimento e Perspectivas da Arborização Urbana no Brasil. In: II Congresso Brasileiro de Arborização Urbana; V Encontro Nacional sobre Arborização Urbana. Anais... São Luiz; SBAU, 1994, p. 15-25.

SCHANZER, H. W. Contribuições da vegetação para o conforto ambiental no câmpus central da PUCRS. Dissertação (Mestrado em Engenharia Civil - Programa de Pós-Graduação em Engenharia Civil, UFRGS, Porto Alegre, 2003.

SHAMS, J. C. A.; GIACOMELI, D. C.; SUCOMINE, N. M. Emprego da arborização na melhoria do conforto térmico nos espaços livres públicos. REVSBAU, Piracicaba - SP, v.4, n.4, p.1-16, 2009 
SOBRAL, M. ; JARENKOW, J. A.; BRACK, P.; IRGANG, B.; LAROCCA, J.; RODRIGUES, R. S. Flora arbórea e arborescente do Rio Grande do Sul, Brasil. $2^{\mathrm{a}}$ ed. São Carlos: RiMa, 2013.

SOMMER, R. Espaço pessoal: as bases comportamentais de projetos e planejamentos. São Paulo, EPU:EDUSP, 1973. 